\title{
Corrigendum
}

\section{Survey of Living Environmental Land Contaminated with Radioactive Materials due to Fukushima Daiichi Nuclear Power Plant Accident}

\section{Sin-ya HOHARA*, Masayo INAGAKI, Kiyoshi KOJIMA, Hirokuni YAMANISHI, Genichiro WAKABAYASHI, Wataru SUGIYAMA and Tetsuo ITOH}

Correction of Table 1 in page 4 has been made as shown below by author declaration.

Table 1 Specific activity of soil samples

\begin{tabular}{cccccc}
\hline Nuclide & $\begin{array}{c}\text { Half-life } \\
{[\text { day }]}\end{array}$ & $\begin{array}{c}\text { Nasushiobara City } \\
\text { Kuroiso Park } \\
{[\mathrm{Bq} / \mathrm{kg}]}\end{array}$ & $\begin{array}{c}\text { Koriyama City } \\
\text { Hayama Park } \\
{[\mathrm{Bq} / \mathrm{kg}]}\end{array}$ & $\begin{array}{c}\text { Fukushima City } \\
\text { Arakawa Athletic } \\
\text { Park }[\mathrm{Bq} / \mathrm{kg}]\end{array}$ & $\begin{array}{c}\text { Regulation } \\
\text { level* }[\mathrm{Bq} / \mathrm{kg}]\end{array}$ \\
\hline${ }^{95} \mathrm{Nb}$ & 35 & $3.26 \mathrm{E} 1 \pm 0.91 \mathrm{E} 1$ & $8.33 \mathrm{E} 1 \pm 1.79 \mathrm{E} 1$ & $1.26 \mathrm{E} 2 \pm 0.28 \mathrm{E} 2$ & $1.0 \mathrm{E} 4$ \\
${ }^{131} \mathrm{I}$ & 8 & $6.49 \mathrm{E} 2 \pm 0.19 \mathrm{E} 2$ & $4.25 \mathrm{E} 3 \pm 0.04 \mathrm{E} 3$ & $2.21 \mathrm{E} 4 \pm 0.01 \mathrm{E} 4$ & $1.0 \mathrm{E} 5$ \\
${ }^{129} \mathrm{Te}$ & 0.048 & $2.31 \mathrm{E} 3 \pm 0.21 \mathrm{E} 3$ & $6.84 \mathrm{E} 3 \pm 0.41 \mathrm{E} 3$ & $2.34 \mathrm{E} 4 \pm 0.07 \mathrm{E} 4$ & $1.0 \mathrm{E} 5$ \\
${ }^{129 \mathrm{~m}} \mathrm{Te}$ & 34 & $3.05 \mathrm{E} 3 \pm 0.32 \mathrm{E} 3$ & $1.06 \mathrm{E} 4 \pm 0.07 \mathrm{E} 4$ & $3.67 \mathrm{E} 4 \pm 0.11 \mathrm{E} 4$ & $1.0 \mathrm{E} 6$ \\
${ }^{132} \mathrm{Te}$ & 3 & $2.98 \mathrm{E} 1 \pm 0.92 \mathrm{E} 1$ & $1.09 \mathrm{E} 2 \pm 0.19 \mathrm{E} 2$ & $2.76 \mathrm{E} 2 \pm 0.34 \mathrm{E} 2$ & $1.0 \mathrm{E} 5$ \\
${ }^{134} \mathrm{Cs}$ & 753 & $4.83 \mathrm{E} 3 \pm 0.04 \mathrm{E} 3$ & $2.11 \mathrm{E} 4 \pm 0.01 \mathrm{E} 4$ & $5.18 \mathrm{E} 4 \pm 0.01 \mathrm{E} 4$ & $1.0 \mathrm{E} 4$ \\
${ }^{136} \mathrm{Cs}$ & 13 & $1.74 \mathrm{E} 2 \pm 0.11 \mathrm{E} 2$ & $6.99 \mathrm{E} 2 \pm 0.22 \mathrm{E} 2$ & $1.80 \mathrm{E} 3 \pm 0.04 \mathrm{E} 3$ & $1.0 \mathrm{E} 4$ \\
${ }^{137} \mathrm{Cs}$ & 11020 & $5.10 \mathrm{E} 3 \pm 0.05 \mathrm{E} 3$ & $2.24 \mathrm{E} 4 \pm 0.01 \mathrm{E} 4$ & $5.50 \mathrm{E} 4 \pm 0.02 \mathrm{E} 4$ & $1.0 \mathrm{E} 4$ \\
\hline
\end{tabular}

* minimum specific activity stipulated for handling in a radiation controlled area under Japanese regulation ${ }^{14)}$.

\footnotetext{
* Corresponding author, E-mail: hohara@kindai.ac.jp DOI : 10.15669/fukushimainsights. Vol.4.169

(C) 2021 Atomic Energy Society of Japan. All rights reserved.

Originally published in Transactions of the Atomic Energy Society of Japan (ISSN 1347-2879), Vol. 11, No. 4, p.340 (2012)

in Japanese.
} 